\title{
Habilidades sociais infantis: comparações por gênero, idade e características escolares
}

\section{Children's social skills: comparison by gender, age and school characteristics}

\section{Habilidades sociales de niños: comparación por género, edad y características escolares}

\author{
Thaciana Araujo da Silva* \\ Universidade Federal do Pará - UFPA, Pará, Brasil \\ Lília I êda Chaves Cavalcante** \\ Universidade Federal do Pará - UFPA, Pará, Brasil
}

\begin{abstract}
RESUMO
Habilidades sociais podem variar ao longo do desenvolvimento do indivíduo em razão de características pessoais e escolares. Neste estudo verificou se há diferenças nas médias de habilidades sociais em dois grupos de crianças de acordo com gênero, idade, ano escolar, presença de dificuldades de aprendizagem e problemas de comportamento. Participaram 81 crianças, entre seis e doze anos, alunos do segundo ao quinto ano do Ensino Fundamental e nove professores. Utilizou-se o Inventário Multimídia de Habilidades Sociais de Crianças - Del Prette (IMHSC-Del Prette). Os resultados indicaram que crianças com idades entre seis e oito anos e alunos no segundo ano escolar, são propensas a apresentar médias inferiores de habilidades sociais em relação àquelas entre nove a doze, que cursavam o terceiro ao quinto ano. Não houve diferenças significativas para dificuldades de aprendizagem e problemas de comportamento. Aponta-se que idade e ano escolar foram preditivos para presença de baixas habilidades sociais na amostra investigada, entretanto ressalta-se a necessidade de conhecer características do contexto de desenvolvimento das crianças por entender que padrões culturais de relações influenciam na presença ou ausência de adequado repertório social. Outras medidas de avaliação comportamental devem ser aplicadas em pesquisas futuras no contexto pesquisado.
\end{abstract}

Palavras-chave: habilidades sociais, crianças, escolares.

\begin{abstract}
Social skills can vary over an individual's development because of personal and school characteristics. This study examined whether there are differences in means between social skill in two groups of children according to gender, age, school year, the presence of learning disabilities and behavior problems. Participants included 81 children between the ages of six and twelve, elementary school students and nine teachers. The Multimedia Social Skills Inventory of Children- Del Prette (IMHSC-Del Prette) was used. The results indicated that children between the ages of six and eight years
\end{abstract}


old and students in the second year of school are likely to present lower mean social skills compared to those between nine and twelve, who were in the third to fifth school year. There were no significant differences for learning difficulties and behavior problems. It is pointed out that age and school year were predictive of the presence of low social skills for the sample investigated, however it is necessary to know the characteristics of the children's development context because it's understand that cultural patterns of relationships influence the presence or absence of adequate social repertoire. Other measures of behavioral evaluation should be applied in future research in the researched context.

Keywords: social skills, children, students.

\section{RESUMEN}

Las habilidades sociales pueden variar a lo largo del desarrollo de un individuo debido a las características personales y escolares. Este estudio examinó si existen diferencias en las medias de las habilidades sociales en dos grupos de niños de acuerdo al sexo, edad, año escolar, presencia de dificultades de aprendizaje y problemas de conducta. Participaron 81 niños, entre seis y doce años, estudiantes del quinto año de la escuela primaria y nueve profesores. Se utilizó el Inventario Multimedia de Habilidades Sociales de Niños-Del Prette (IMHSC-Del Prette). Los resultados indicaron que los niños de seis y ocho años, y cursando el segundo año de la escuela, es probable que tengan medias más bajas de habilidades sociales en comparación con los de nueve a doce, que estaban en el tercer al quinto año. No hubo diferencias significativas en las dificultades de aprendizaje y problemas de conducta. Señala que la edad y el año escolar son predictivos de la presencia de bajas habilidades sociales en la muestra investigada, sin embargo, hace la necesidad de conocer las características del desarrollo de los niños del contexto por entender que los patrones culturales de las relaciones influyen en la presencia o ausencia de adecuados repertorio social. Otras medidas de evaluación del comportamiento se deben aplicar en el futuro en la investigación en este contexto.

Palabras clave: habilidades sociales, niños, estudiantes.

\section{I ntrodução}

As pesquisas que descrevem e classificam as habilidades sociais de indivíduos e grupos, assim como os programas de treinamento para a aquisição de repertórios comportamentais têm evidenciado a importância de se conhecer de forma contextualizada essas habilidades por constituírem-se importantes aspectos do desenvolvimento humano. O termo habilidades sociais pode ser definido por um conjunto de diferentes classes de comportamentos sociais do repertório de um indivíduo, os quais contribuem para competência social e favorecem um relacionamento saudável e produtivo com as demais pessoas (Del Prette \& Del Prette, 2009). A competência social, por sua vez, é a capacidade de articular sentimentos, pensamentos e ações em função de objetivos pessoais e de demandas da situação e da cultura, gerando consequências positivas para o próprio indivíduo e para a sua relação com as outras 
pessoas (Del Prette \& Del Prette, 2009; Gresham, 2009). Segundo Caballo (2012), o comportamento socialmente hábil é aquele que engloba um conjunto de comportamentos emitidos pelo indivíduo, em um contexto interpessoal, que expressa atitudes, sentimentos, desejos e opiniões ou direitos, de forma adequada às situações colocadas no convívio social, tornando-o eficaz na resolução de problemas imediatos, minimizando a probabilidade de problemas futuros.

Pode-se entender as habilidades sociais no centro de um contínuo em que, de um lado, estão reações não-habilidosas passivas que são comportamentos que se expressam de forma encoberta, tais como ansiedade, mágoa, ressentimento e/ou esquiva ou fuga de demandas interpessoais e em situações conflituosas, e, de outro, estão as reações não-habilidosas ativas que são comportamentos que se expressam de forma aberta, como agressividade verbal ou física, ironia e coerção (Del Prette \& Del Prette, 2005). Além disso, admitese que as habilidades sociais da pessoa em desenvolvimento e do seu contexto relacional e cultural atuam conjuntamente exercendo influência sobre o seu repertório social em formação (Del Prette \& Del Prette, 2009). Por exemplo, a variável gênero tem sido associada a um repertório adequado de habilidades sociais, como indicam os estudos de Fumo (2009), Garaigordobil e Maganto (2011) e Valle e Garnica (2009).

Segundo a Teoria Bioecológica do Desenvolvimento Humano (Bronfenbrenner 1996, 2011), características biopsicológicas da pessoa, tais como idade, gênero, etnia, e aparência física, sofrem interferências de valores, crenças e papéis sociais que permeiam o contexto que a pessoa interage (Leme, Del Prette, Koller \& A. Del Prette, 2015). Assim, pesquisas evidenciaram a predominância de reações não-habilidosas passivas e maiores médias de reações habilidosas em crianças do gênero feminino, enquanto que em relação ao gênero masculino foram observadas mais frequentemente reações não-habilidosas ativas.

Nessas pesquisas, foram utilizados por Fumo (2009), Valle e Garnica (2009), Garaigordobil e Maganto (2011) o Social Skills Rating System (SSRS) e o IMHS-Del Prette, respectivamente, além de outros instrumentos para a avaliação de empatia, atitudes sobre conflitos e estilos adotados para sua resolução. Na mesma direção, o estudo longitudinal de Pizato, Marturano e Fontaine (2014) encontrou também diferenças dos valores de habilidades sociais, sendo as meninas classificadas como mais habilidosas socialmente que os meninos, quando em idade escolar, e foram avaliadas pelo SSRSversão professor, ao longo de três anos.

As diferenças entre as médias de habilidades sociais de crianças e outras categorias de participantes de acordo com a sua condição de gênero, levam em consideração fatores culturais dos ambientes 
principais em que a criança se desenvolve, como a família e a escola, pois as relações estabelecidas nesses meios interferem nas expectativas sociais de desempenho de papéis colocados aos meninos e às meninas (Abdi, 2010; Anme et al.,2010; Caballo, 2012).

Outra característica pessoal relacionada às habilidades sociais é a idade (Bolsoni-Silva, Marturano \& Freiria, 2010; Garaigordobil \& Maganto, 2011; Sabol \& Pianta, 2011; Welsh et al, 2001). Tais estudos mostram que crianças em idade pré-escolar (4 e 5 anos) apresentam menos habilidades sociais quando comparadas a amostras de escolares e adolescentes, apontando que ganhos no repertório socialmente habilidoso se acentuam conforme 0 amadurecimento psicológico e da autorregulação emocional da criança (Bolsoni-Silva et al., 2010; Montroy, Bowles, Skibbe \& Foster, 2014).

No que diz respeito às características escolares que podem afetar as habilidades sociais encontram-se desempenho acadêmico e dificuldades de aprendizagem (Arnold et al., 2012; Feitosa, Del Prette, Del Prette, \& Loureiro, 2011; Fram, Kim, \& Sinha, 2012) que contribuem para a discussão existente na educação sobre o fracasso escolar (Del Prette \& Del Prette, 2003). Crianças com dificuldades socioemocionais com menor repertório de habilidades sociais, sendo passivas ou agressivas nas suas relações com colegas de turma e com professores, apresentam muitas vezes dificuldades de aprendizagem, pois deixam de emitir comportamentos apropriados ao processo de ensino-aprendizagem durante as aulas. Como exemplo, citam-se os seguintes comportamentos: orientar-se para a tarefa, ignorando interrupções; participar nas discussões em classe; seguir regras ou instruções orais; observar e prestar atenção; aguardar a vez para falar; oferecer, solicitar e agradecer ajuda; fazer e responder perguntas (Del Prette \& Del Prette, 2005, 2009).

Assim, estudos com variáveis escolares feitos por Barreto, Freitas e Del Prette (2011) e Feitosa et al. (2011) procuraram avaliar a relação entre dificuldades de aprendizagem e desempenho acadêmico com o repertório de habilidades sociais observado, concluindo que a presença de repertório social adequado tem relações com 0 desempenho escolar de crianças, ou seja, quanto menores as dificuldades de aprendizagem e de desempenho acadêmico apresentados, maiores as habilidades sociais. Um estudo longitudinal que acompanhou, por nove anos crianças da pré-escola ao oitavo ano, conduzido por Caemmerer e Kaith (2015), investigou se as habilidades sociais produziram efeito no desempenho acadêmico ou se o desempenho acadêmico é que teria impacto sobre as habilidades sociais. Esse estudo encontrou nos modelos testados que há reciprocidade dos efeitos entre habilidades sociais e desempenho acadêmico (leitura e matemática) em crianças acompanhadas no período escolar por nove anos. Os autores apontam que uma das 
habilidades que os estudantes podem ter desenvolvido seriam as habilidades de resolução de conflitos que tem uma relação direta na qualidade de interação entre pares e professores e favorece um apoio escolar. A relação de altas habilidades sociais e baixo desempenho acadêmico ocorre no sentido de que a criança emita comportamentos que favorecem sua aprendizagem e ter interações positivas com seus pares e professores, como por exemplo, realizar perguntas quando possui dúvida, seguir regras e ajudar o colega na tarefa (Del Prette \& Del Prette, 2005, 2009).

Outra questão investigada nesta área de pesquisa são as possíveis associações entre habilidades sociais e problemas de comportamento, expressos tanto de forma externalizante quanto internalizante (Bolsoni-Silva et al., 2010; Feitosa et al., 2011; Casali-Robalinho, Del Prette \& Del Prette, 2015). Esses estudos indicaram a presença predominante de correlações negativas, isto é, verificou-se que quanto menor a média de habilidades sociais maior a incidência de problemas de comportamento. O estudo longitudinal de Pizato et al. (2014) apontou tendência ao aumento das médias obtidas ao longo dos três anos escolares pesquisados. Ou seja, aumentaram as habilidades sociais e diminuíram os problemas de comportamento entre os escolares com o avanço dos anos. Pelo exposto, parece haver uma estreita relação entre medidas de desempenho social e variáveis escolares, tais como habilidades de leitura e escrita (Molina \& Del Prette, 2006; Sabol \& Pianta, 2011), dificuldades de aprendizagem (Barreto et al., 2011; Feitosa et al., 2011) e problemas de comportamento (Bolsoni-Silva et al., 2010; Casali-Robalinho et al., 2015; Feitosa et al., 2011; Pizato et al., 2014).

Entretanto, especificamente em relação ao ano escolar, os estudos levantados não têm sido tão conclusivos quanto o esperado, sobretudo quando envolveram crianças no Ensino Fundamental, que neste caso os estudos citados ainda usavam a nomenclatura "série" para se referir ao "ano escolar", utilizado hoje. Saud e Tonelotto (2005), por exemplo, indicaram que problemas de relacionamento com colegas e a ocorrência de comportamentos pró-sociais de classificação 'normal' foram predominantes em escolares da 3ạ série (equivalente ao 2 o ano escolar). Por sua vez, Bandeira et al. (2006) notaram que comportamentos considerados problemáticos tornaramse menos frequente ao longo das séries escolares, sendo mais evidentes na $1^{\mathrm{a}}$ série ( 20 ano) e menos comuns na $4^{\mathrm{a}}$ série ( $\left.5^{\circ} \mathrm{ano}\right)$. Do mesmo modo, quanto maior era o grau de dificuldades de aprendizagem pela criança, mais alta era a frequência de comportamentos problemáticos e menores as habilidades sociais observadas. A despeito disso, Fumo (2009) não encontrou diferença significativa entre os anos escolares em relação às habilidades sociais ao comparar grupos de crianças de 1 à à 4 a série divididos em grupos de baixo e alto desempenho escolar classificados pelos professores. 
Já Pizato et al. (2014) ao verificarem correlações entre habilidades sociais e ano escolar, encontraram maiores médias entre o 3 ㅇ e 5 은 ano no mesmo grupo de crianças.

As discordâncias destacadas nos estudos de Bandeira et al. (2006), Fumo (2009), Pizato et al. (2014) e Saud e Tonelotto (2005), sugerem que o repertório de habilidades sociais pode variar conforme a série cursada, porém, o quanto essa diferença será significativa requer investigações sistemáticas e contextualizadas para a sua compreensão. Supõe-se que cada série tenha suas particularidades em termos de atividades e conteúdos ensinados, e que a dinâmica em sala de aula pode interferir na qualidade das relações estabelecidas, uma vez que cada série é um novo contexto ecológico de desenvolvimento que exigem ajustes e ampliações de desenvolvimento de atividades, papéis e relações da criança (Bronfenbrenner, 1996). Além disso, atenta-se que no delineamento dos estudos apresentados, embora concordem em alguns resultados, as formas como as variáveis escolares foram avaliadas com diferentes instrumentos e informantes (professores, crianças) são fatores que podem alterar de forma substancial os resultados de pesquisas realizadas com uma mesma população, por exemplo (Dinnebeil et al., 2013).

Assim, considerando as possibilidades de papéis, atividades, e relações que a escola oferece às crianças, percebe-se que este contexto é fundamental não só para transmissão de conteúdos disciplinares como por se constituir em um espaço de aprimoramento de melhorias nas relações interpessoais. Na visão da bioecologia do desenvolvimento humano (Bronfenbrenner, 2011), o ambiente escolar deve ser entendido como um microssistema que possibilita a ampliação de experiências afetivas e a aquisição de conhecimentos, entre outras razões, posto ser este ambiente um legítimo representante da cultura da sociedade vivida (Marturano \& Loureiro, 2007). Por isso, pesquisadores da área (Arnold et al., 2012; Ashdown \& Bernard, 2012; Arslan, Durmusoglu-Saltali, \& Yilmaz, 2011; Dereli, 2009; Moreira et al., 2010; Moreira et al, 2014) têm investido em programas de intervenção para avaliação e promoção de habilidades sociais em crianças, principalmente entre pré-escolares. Nesse sentido, destaca-se a importância da participação de professores para a prevenção de conflitos entre pares e melhorias no desempenho acadêmico e na qualidade de relações interpessoais, por exemplo, Sabol e Pianta (2011) e Molina e Del Prette (2006) identificaram melhorias em aquisições acadêmicas (leitura e escrita) em grupos de crianças que participaram de projeto de promoção de habilidades.

Os resultados desses estudos têm encontrado desdobramentos positivos na expansão do repertório socialmente habilidoso das crianças, além de diminuição de problemas de comportamento entre pares a partir da sua exposição a programas de intervenção no 
contexto escolar, sobretudo em áreas como autocontrole, empatia, assertividade, regulação emocional e estratégias de coping.

Outrossim, parece existir uma preocupação comum entre pesquisadores da área na atualidade (Bolsoni-Silva et al., 2010; Dereli, 2009; Sabol \& Pianta, 2011): é preciso conhecer as habilidades sociais infantis de forma sistemática e contextualizada, já que quanto mais cedo forem detectados possíveis déficits nesse importante domínio do desenvolvimento, maiores são as chances de prevenir consequências negativas para os relacionamentos sociais que a criança irá construir nos primeiros anos e ao longo da vida. Por isso, torna-se importante compreender as habilidades sociais em sua complexa ecologia, procurando identificar o quanto características pessoais e contextuais, com destaque para variáveis relativas ao ambiente escolar, estariam associadas à presença de um repertório socialmente habilidoso na infância.

Neste sentido, a partir do que foi apresentado sobre a relação entre habilidades sociais e aspectos da pessoa e características escolares, uma forma de investigar possíveis diferenças no repertório social de crianças de diferentes contextos são os estudos transversais. No caso do Brasil, os estudos de avaliação de habilidades sociais na região Norte ainda não são expressivos, sendo importante conhecer se em Belém, por sua importância política, social e cultural na região, as crianças seguem essa tendência nacional e internacional, uma vez que o contexto é um fator importante para a compreensão do desenvolvimento de habilidades sociais.

Desta maneira, considerando a importância de características pessoais e variáveis escolares no repertório de habilidades sociais para o desenvolvimento da criança, o objetivo deste artigo foi verificar se há diferença entre as médias obtidas por dois grupos de crianças formados por alunos do segundo ao quinto ano do Ensino Fundamental na cidade de Belém-PA, em relação ao gênero, idade, ano escolar, dificuldades de aprendizagem, e problemas de comportamento.

Este trabalho foi orientado na perspectiva do Modelo Bioecológico do Desenvolvimento Humano na medida em que investigou possíveis associações entre variáveis pessoais e contextuais e as habilidades sociais infantis avaliadas, procurando demonstrar se os resultados seguem tendências nacional e internacional apontadas na literatura PSI e quais as particularidades desse quadro em uma região do país onde este tipo de investigação tem sido ainda pouco estudado. Considerando-se que escolares do sexo masculino, mais jovens, com dificuldades de aprendizagem e problemas de comportamento, tendem a apresentar menores médias de habilidades sociais em relação aos do sexo feminino, com mais idade, sem dificuldades de aprendizagem e problemas de comportamento, pretende-se investigar o quanto esse é um pressuposto válido para alavancar 
estudos na região Norte do Brasil e orientar futuros projetos de intervenção para promoção de habilidades.

\section{Método}

Participantes

Participaram 81 crianças (40 meninos; 41 meninas), com idade entre 6 a 12 anos, alunos do segundo ao quinto ano escolar, e nove professores vinculados a uma escola de ensino fundamental de Belém-PA, no Norte do Brasil. Apenas uma criança de seis anos participou, pois ela estava às vésperas de completar 7 anos. Os participantes foram selecionados aleatoriamente a partir de um universo amostral composto por 109 alunos e, em seguida, organizados em dois grupos, conforme as médias de reações habilidosas e não-habilidosas obtidas por um instrumento destinado à avaliação de habilidades sociais infantis.

Instrumentos

Utilizou-se o Inventário Multimídia de Avaliação de Habilidades Sociais de Crianças - versão impressa (IMHSC-Del Prette), elaborado por Del Prette e Del Prette (2005), que avalia o repertório de habilidades sociais a partir dos indicadores de frequência, adequação e dificuldade de emissão de reações habilidosas de crianças de 7 a 12 anos. Neste estudo foi utilizada somente a versão de autoavaliação e o indicador de frequência ( sempre $=2$, às vezes $=1$, nunca $=0$ ) das reações habilidosas e não-habilidosas, cabendo aos professores que indicassem na ficha de cada criança a presença de dificuldades de aprendizagem (muita, pouca ou nenhuma), assim como de problemas de comportamento (muito, pouco ou nenhum). Este instrumento possui consistência interna aferida pelo alfa de Cronbach de 0,64 para frequência conforme descrito no manual e, para a amostra deste estudo, o alfa foi de 0,05.

Procedimento de coleta de dados

A coleta de dados realizou-se em uma instituição de ensino de cunho filantrópico, mas que possui convênio com órgãos governamentais, que atende crianças de creche, pré-escola até o quinto ano do Ensino Fundamental. As etapas da pesquisa consistiram em apresentar 0 projeto à direção da escola assim como sua autorização. O projeto foi submetido para avaliação no comitê de Ética e, após sua aprovação, foi dado início à coleta de dados. Foi enviada aos cuidadores (responsáveis pelas crianças) uma carta-convite para participarem da 
pesquisa e aqueles que aceitaram assinaram o Termo de Consentimento Livre e Esclarecido (TCLE).

A coleta com as crianças através do IMHSC-Del Prette, aplicada em dupla de crianças, ocorreu em duas sessões de, aproximadamente, 40 minutos. O local de aplicação foi uma sala isolada de ruídos com mesas e cadeiras individuais para cada criança e a pesquisadora apresentava o caderno de questões e solicitava que elas respondessem. As duplas foram selecionadas por ordem alfabética conforme a chamada. Foi feita uma instrução clara para as crianças a fim de que durante a aplicação fossem evitadas conversas ou comportamentos que prejudicassem o preenchimento da ficha, e eram lembradas sempre que necessário, entretanto as crianças mostraram-se interessadas em participar. Após essa etapa, os professores foram convidados a preencherem na própria ficha de avaliação do IMHSC-Del Prette no espaço próprio destinado, se havia presença de dificuldades de aprendizagem (muita, pouca, nenhuma) e problemas de comportamento (muito, pouco, nenhum) de cada criança participante.

Análise de dados

Para efeito das análises pretendidas, foram consideradas duas amostras neste estudo: O Grupo A $(n=33)$, constituído de crianças consideradas habilidosas socialmente, foi formado por participantes que apresentaram simultaneamente "altas médias de reações habilidosas", "baixas médias de reações não-habilidosas ativas" e "não-habilidosas passivas". O Grupo B ( $n=48)$, grupo avaliado como não-habilidoso socialmente, foi composto por crianças que apresentavam as características opostas: "baixas médias de reações habilidosas", "altas médias de reações não-habilidosas ativas" e "nãohabilidosas passivas". A classificação das crianças conforme as médias das reações habilidosas, não-habilidosas ativas e nãohabilidosas passivas foi feita considerando-se como ponto de corte a região do desvio interquartílico (entre Percentil 25 e Percentil 75). Desta forma, as crianças indicadas como tendo baixa pontuação obtiveram médias menores que o Primeiro Quartil (P25). As crianças com altas pontuações obtiveram médias maiores que o Terceiro Quartil (P75), chegando-se ao total de 81 crianças. Desse modo, as crianças classificadas como pertencentes ao Grupo A (13 meninos, 20 meninas; média de idade 9 anos), portanto, habilidosas socialmente, foram as que obtiveram valores médios na faixa entre 1,62 e 2,0, enquanto que aquelas classificadas como não habilidosas, constituíram o Grupo B (27 meninos, 21 meninas; média de idade 8 anos), e apresentaram valores médios na faixa entre 0,00 e 1,29.

Os dados dos professores foram agrupados em presente (muita ou pouca dificuldade de aprendizagem; muito ou pouco problemas de 
comportamento) ou ausente (nenhuma dificuldade de aprendizagem ou nenhum problema de comportamento). Em seguida, esses dados e as variáveis gênero e ano escolar foram comparados em relação às medidas de habilidades sociais conforme os grupos de crianças com maiores e menores médias de habilidades sociais (Grupo A e Grupo B, respectivamente), utilizando o Teste Qui-Quadrado. O teste $U$ de Mann-Whitney foi utilizado para a comparação dos grupos em relação à variável idade, e foi calculada uma regressão logística a fim de identificar o perfil probabilístico de crianças pertencerem ao grupo B, a partir do programa estatístico BioEstat versão 5.4.

\section{Considerações Éticas}

Esta pesquisa foi aprovada junto ao Comitê de Ética do Núcleo de Medicina Tropical, sob CAAE de número 10796412.6.0000.5172.

\section{Resultados e Discussão}

Os resultados das análises em relação às variáveis gênero, ano escolar, dificuldades de aprendizagem e problemas de comportamento estão indicados na Tabela 1.

Em relação ao ano escolar, as médias de habilidades sociais obtidas pelas crianças do Grupo A e do Grupo B apresentaram diferença significativa $(p=0,0032)$. Encontrou-se que crianças com maiores médias nas habilidades sociais (Grupo A) frequentavam série escolar mais avançada ( 4 o e 5 o ano) o que confirma o estudo realizado por Bandeira et al. (2006) e Pizato et al., (2014), todavia, contraria os resultados de Saud e Tonelotto (2005) que apontaram relação inversa, assim como Fumo (2009) que também não encontrou diferenças no desempenho social entre crianças de diferentes anos escolares. Pode-se pensar que conforme o ano cursado pela criança, as exigências mudam em relação ao papel social de aluno e mais regras e participação nas atividades são exigidas, o que requer um maior controle emocional e domínio de habilidades relacionais, além do crescimento da criança também ocorrer. A atuação em diversos contextos ecológicos exige da pessoa em desenvolvimento 0 desempenho de atividades, papéis e relações progressivamente mais complexos e isso pode contribuir para um desfecho positivo do seu amadurecimento como indivíduo através de ganhos socioemocionais e controle do comportamento (Leme et al., 2015). Entretanto, percebe-se que a variável ano escolar e suas implicações em termos do desenvolvimento demandam investigações que sejam sensíveis às características específicas de cada contexto (Bronfenbrenner, 1996) e também, aponta-se que efeitos emocionais de experiências relacionais anteriores ao ensino fundamental, como por exemplo, 
situações estressoras e de aceitação e rejeição entre pares podem persistir nos anos escolares subsequentes da criança e favorecer ou dificultar o seu desenvolvimento social (Pizato et al., 2014).

Tabela1

Comparação das Habilidades Sociais em relação às Características Pessoais e Escolares nos Grupo A e Grupo B, conforme o Qui-quadrado

\begin{tabular}{|c|c|c|c|c|c|c|}
\hline & & \multicolumn{2}{|c|}{ Grupo A } & \multicolumn{2}{|c|}{ Grupo B } & \multirow[b]{2}{*}{$p$} \\
\hline & & $\mathrm{N}$ & $\%$ & $\mathrm{~N}$ & $\%$ & \\
\hline & Masculino & 13 & 39,4 & 27 & 56,3 & 0,2060 \\
\hline \multirow[t]{2}{*}{ Gênero } & Feminino & 20 & 60,6 & 21 & 43,8 & \\
\hline & $2^{\circ}$ ano & 0 & 0 & 15 & 31,3 & $0,0032^{*}$ \\
\hline \multirow[t]{3}{*}{ Ano escolar } & $3^{\circ}$ ano & 9 & 27,3 & 13 & 27,1 & \\
\hline & $4^{\circ}$ ano & 10 & 30,3 & 8 & 16,7 & \\
\hline & $5^{\circ}$ ano & 14 & 42,4 & 12 & 25,0 & \\
\hline \multirow{3}{*}{$\begin{array}{l}\text { Dificuldade } \\
\text { aprendizagem }\end{array}$} & Ausente & 15 & 45,5 & 20 & 41,7 & 0,9125 \\
\hline & Presente & 18 & 54,5 & 28 & 58,3 & \\
\hline & Ausente & 23 & 69,7 & 28 & 58,3 & 0,4200 \\
\hline Problemas de & Presente & 10 & 30,3 & 20 & 41,7 & \\
\hline Comportamento & & & & & & \\
\hline
\end{tabular}

Apesar do fato de as crianças que pertenceram ao Grupo A, fossem predominantemente do sexo feminino, enquanto que o Grupo $B$ apresentou maior número de participantes do sexo masculino, verifica-se que não houve diferença estatisticamente significativa entre os grupos no que se refere às médias obtidas pelos mesmos. Este resultado destoa da literatura da área que indica que as meninas apresentam, geralmente, melhor repertório social em relação aos meninos (Fumo, 2009; Garaigordobil \& Maganto, 2011; Pizato et al., 2014; Valle \& Garnica, 2009). Este resultado talvez possa ser explicado pela variedade de métodos de pesquisa presente na literatura PSI (Dinnebeil et al., 2013), que, em muitos casos, diferenciam-se do que orientou o presente estudo. Nesse sentido, 
admite-se que essa variação metodológica possa explicar a diferença estatisticamente não significativa entre os grupos de crianças, segundo a variável sexo. I gualmente é importante ressaltar que as habilidades sociais são aprendidas conforme modelos e oportunidades disponíveis no ambiente (Del Prette \& Del Prette, 2009; Leme et al, 2015), sendo capazes de provocar variações no desempenho social de grupos de meninas e meninos quando comparados (Abdi, 2010; Anme et al.,2010; Caballo, 2012).

Sobre problemas de comportamento e dificuldades de aprendizagem estes achados discordam dos da literatura apresentada. Para esta amostra, estas variáveis não variavam conforme maiores ou menores habilidades sociais apresentadas pelas crianças, por exemplo, se maiores médias de reações habilidosas, menos seriam as dificuldades de aprendizagem e problemas de comportamento delas (Bandeira et al., 2006; Barreto et al., 2011; Caemmerer e Kaith, 2015; Feitosa et al., 2011; Pizato et al., 2014). Essas divergências também podem ser em função da forma como as medidas foram coletadas no presente estudo, que contou apenas com a informação dos professores sobre cada criança em relação a essas informações, sem que houvesse um instrumento específico que medisse as dificuldades de aprendizagem e a incidência de presença de problemas de comportamento apresentados pelas crianças participantes. Ressalta-se que diferentes informantes e medidas para avaliação de tais aspectos podem contribuir para maior compreensão das relações com as habilidades sociais das crianças (Dinnebeil et al, 2013). Além disso, o contexto escolar e seus integrantes representam um microssistema de desenvolvimento que deve disponibilizar práticas pedagógicas que atendam às necessidades da criança, facilitando o processo ensinoaprendizagem e desenvolvimento de outras habilidades e competências sociais.

Assim, a própria promoção de habilidades sociais, neste contexto, torna-se importante uma vez que outros estudos (Molina \& Del Prette, 2006; Sabol \& Pianta, 2011) apontam para benefícios na leitura e desempenho acadêmico em crianças com pontuações elevadas na avaliação de habilidades sociais e melhora nos relacionamentos entre pares, com a redução de problemas de comportamentos ao longo das séries, como apontado por estudos longitudinais como o de Moreira et al. (2014).

Outra variável que apresentou diferença significativa ( $p=0,0008)$ entre os grupos foi idade. A distribuição dos dados pessoais pelo Teste $U$ de Mann-Whitney indicou mediana de nove anos nos participantes do Grupo A e de oito anos no Grupo B. Sobre a variável idade, verificou-se que a faixa etária das crianças no Grupo $A$ foi de seis a oito anos, enquanto que os participantes do Grupo B possuíam idades a partir de nove anos de idade. Estes resultados são coerentes com os dados da literatura (Bolsoni-Silva et al, 2010; Garaigordobil \& 
Maganto, 2011; Sabol \& Pianta, 2011; Welsh et al, 2001) que indica haver um aumento gradual no repertório social habilidoso das crianças conforme a sua idade avança, por considerar seu maior amadurecimento psicológico, desenvolvimento de autorregulação emocional e senso de autonomia em relação aos cuidadores primários (Bolsoni-Silva et al., 2010; Montroy et al, 2014).

Conforme o Modelo Bioecológico do Desenvolvimento Humano, as características biopsicológicas sofrem influência do contexto e o influencia, e são expressas ao longo do tempo através de estabilidades e mudanças (Bronfenbrenner, 2011). Neste estudo, o gênero não foi um fator determinante entre os grupos, enquanto a idade mostrou-se importante nesta amostra para diferenciar o repertório de habilidades sociais entre as crianças. A partir do que a literatura aponta, a hipótese deste estudo era encontrar diferenças entre habilidades sociais entre meninos e meninas e em crianças com mais idade, sendo meninas mais velhas consideradas mais habilidosas.

Por meio de análise de regressão logística das variáveis que apresentaram significância estatística, dispostos na Tabela 2, foi possível inferir que em crianças com idade entre seis e oito anos e que cursavam o 2 o ano escolar estavam propensas a pertencerem ao Grupo B ( $g l=3 ; p<0,0001)$.

Tabela 2

Regressão logistica de variáveis idade, relacionamento e ano escolar

\begin{tabular}{llll}
\hline & Coeficientes & Variáveis preditoras & Probabilidade de Grupo B \\
Beta 1 & 0,6351 & Idade entre 6 e 8 anos & $\mathrm{p}=0,1943$ (ou 19,43\%) \\
Beta 4 & 10,8210 & Estar no $2^{\circ}$ ano escolar & $\mathrm{p}=0,9998($ ou $99,98 \%)$ \\
\hline
\end{tabular}

Nota. $\mathrm{p}<0,0001$

A partir da regressão logística obteve-se que crianças que cursam o segundo ano e possuem idades entre 6 a 8 anos possuem baixas médias de habilidades sociais, ou seja, altos índices de reações nãohabilidosas ativas e passivas. Os efeitos dos seus atributos têm efeitos esperados sobre sua trajetória desenvolvimental, mas depende provavelmente do grau significativo dos padrões correspondentes de resposta que eles podem evocar do ambiente da pessoa (Bronfenbrenner, 2011). Isso quer dizer que a qualidade da relação estabelecida com as crianças, em determinado contexto e em determinada idade, afeta o curso do desenvolvimento, e que envolvem também os aspectos subjetivos envolvidos nessas interações. Portanto, deve-se considerar que as relações estabelecidas entre os pares afetam a emissão comportamentais 
sociais e que podem ser modificados e aprimorados através de treinos de habilidades sociais para as crianças (Arnold et al., 2012; Ashdown \& Bernard, 2012; Arslan et al, 2011; Leme et al., 2015; Moreira et al, 2014).

\section{Considerações finais}

Este estudo comparou dois grupos de crianças com repertório socialmente habilidoso e não-habilidoso, conforme o IMHSC-Del Prette em relação a aspectos pessoais e escolares. Os resultados indicaram diferenças significativas no que se refere a variáveis como idade e ano escolar, identificando o perfil probabilístico de crianças entre seis a oito anos, estudantes do segundo ano do Ensino Fundamental de apresentarem baixas médias de habilidades sociais.

Aspectos que traduzem características pessoais das quais depende o aprendizado e emissão das habilidades sociais podem explicar as similaridades nos aspectos relacionais, já que individualidades interagem com e no contexto da criança que é dotado de objetos, símbolos e pessoas que também agem no ambiente e nas características de força, demanda e recursos das crianças, conforme afirmam Bronfenbrenner $(1996,2011)$ e Del Prette \& Del Prette (2009).

Sobre o aspecto escolar, este estudo confirmou resultados da literatura no que diz respeito ao repertório de habilidades sociais inadequado em crianças que frequentam séries iniciais, neste caso, crianças do segundo ano e que possuem idades entre 6 a 8 anos. Estas crianças estão numa fase de desenvolvimento em que, frequentemente, ainda necessitam de outras pessoas adultas para haver o apaziguamento dos conflitos com pares ou que apresentam estratégias de resolução de conflitos ineficientes, sendo passivas ou agressivas.

Uma limitação deste estudo que deve ser apontada diz respeito à ausência de medidas de avaliação que gerassem um perfil comportamental da criança, já que o instrumento utilizado foi autoavaliação de habilidades sociais. Esta forma de avaliação, embora utilizada na literatura pertinente e com resultados significativos, pode gerar vieses, visto que é baseada no relato e autopercepção que a pessoa tem do seu comportamento e não do observado em situação natural (Bandeira et al, 2006), além de poder haver falseamento das respostas para informações socialmente aceitas (Barreto et al., 2011; Del Prette \& Del Prette, 2005). Por isso, há necessidade de outros instrumentos de medidas para maior compreensão da variável investigada para confrontar os resultados.

Espera-se que os resultados aqui encontrados sejam úteis na ampliação do olhar sobre habilidades sociais não apenas por 
considerar características da vida escolar da criança que já é amplamente estudada, mas também de características da pessoa e do processo que facilitam ou dificultam a emissão e aprendizagem das habilidades sociais, considerando, para tanto, a passagem do tempo e o contexto em que a criança vive e se relaciona. Isso significa observar os elementos de atividades, papéis e relações, pois dependendo de com quem interagem nos seus ambientes primários (escola e família), da sua complexidade e regularidade podem influenciar positivamente 0 desenvolvimento da criança não só socialmente, mas em outros aspectos do desenvolvimento como o afetivo, por exemplo.

Portanto, na área das habilidades sociais é interessante que haja uma avaliação multimodal para compreender melhor os contextos e fatores relacionados à aquisição, desenvolvimento e manutenção do repertório de habilidades sociais em crianças. Assim, sugerem-se pesquisas que busquem outras formas de análises do fenômeno investigado.

Uma outra sugestão para pesquisas futuras é acompanhar longitudinalmente as crianças para verificar como se comportam as variáveis ao longo do desenvolvimento da criança verificando relações com as características pessoais e escolares investigadas.

\section{Referências}

Abdi, B. (2010). Gender diferences in social skills, problem behaviours and academic competence of I ranian kindergarten children based on their parent and teacher ratings. Procedia Social and Behavioral Sciences, 1175-1179. doi: 10.1016/j.sbspro.2010.07.256

Anme, T., Shinohara, R., Sugisawa Y., Tong, L. Tanaka, E., Watanabe, T., O. et al. (2010). Gender diferences of children's social skills and parentig using Interaction Rating Scale (IRS). Procedia Social and Behavioral Sciences, 2, 260-268. doi: 10.1016/j.sbspro.2010.03.008

Arnold, D. H., Kupersmidt, J. B., Voegler-Lee, M. E., \& Marshall, N. A. (2012). The association between preschool children's social functioning and their emergent academic skills. Early Childhood Research Quarterly, 27(3), 376-386. doi: 10.1016/j.ecresq.2011.12.009

Ashdown, D. M., \& Bernard, M. E. (2012). Can explicit instruction in social and emotional learning skills benefit the social-emotional development, well-being, and academic achievement of young children? Early Childhood Education Journal. 39(6), 397-405. doi: $10.1007 / \mathrm{s} 10643-011-0481-x$ 
Arslan, E., Durmusoglu-Saltali, N., \& Yilmaz, H. (2011). Social skills and emotional and behavioral traits of preschool children. Social Behavior and Personality, 39(9), 1281-1288. doi: 102224/sbp.2011.39.91281

Bandeira, M., Rocha, S. S., Freitas, L. C., Del Prette, Z. A. P., \& Del Prette, A. (2006). Habilidades sociais e variáveis sociodemográficas em estudantes do ensino fundamental. Psicologia em estudo, 11(3), 541-549. doi: 10.1590/S141373722006000300010

Barreto, S. O., Freitas, L. C., \& Del Prette, Z. A. P. (2011). Habilidades sociais na comorbidade entre dificuldades de aprendizagem e problemas de comportamento: Uma avaliação multimodal. PSICO, 4(42), 503-510. doi: 10.15448/19808623.2011.4.7593

Bolsoni-Silva, A. T., Marturano, E. M., \& Freiria, L. R. B. (2010). Indicativos de problemas de comportamento e de habilidades sociais em crianças: Um estudo longitudinal. Psicologia: Reflexão e Crítica, 23(3), 506-515. doi: 10.1590/S010279722010000300011

Bronfenbrenner, U. (1996). A ecologia do desenvolvimento humano: Experimentos naturais e planejados (M. A. V. Veronese, Trad.). Porto Alegre: ArtMed.

Bronfenbrenner, U. (2011). Bioecologia do desenvolvimento humano: Tornando os seres humanos mais humanos (A. CarvalhoBarreto, Trad.). Porto Alegre: Artemed.

Caballo, V. E. (2012). Manual de avaliação e Treinamento das habilidades sociais. São Paulo: Santos.

Caemmerer, J. M., \& Keith, T. Z. (2015). Longitudinal, reciprocal effects of social skills and achievement from kindergarten to eighth grade. Journal of School Psychology, 53, 265-281. doi: 10.1016/j.jsp.2015.05.001

Casali-Robalinho, I. G., Del Prette, Z. A. P., \& Del Prette, A. (2015). Habilidades Sociais como preditoras de problemas de comportamento em escolares. Psicologia: Teoria e Pesquisa, 31(3), 321-330. doi: 10.1590/0102-37722015032110321330

Del Prette, Z.A.P., \& Del Prette, A. (2003). Habilidades sociais e dificuldades de aprendizagem: Teoria e pesquisa sob um enfoque multimodal. In A. Del Prette \& Z.A.P. Del Prette (Orgs.), Habilidades sociais, desenvolvimento e aprendizagem (pp.167206). Campinas, SP: Alínea.

Del Prette, Z. A. P., \& Del Prette, A. (2005). Sistema multimídia de habilidades sociais de crianças (SMHSC-Del-Prette) manual. São Paulo: Casa do Psicólogo.

Del Prette, Z. A. P., \& Del Prette, A. (orgs.). (2009). Psicologia das habilidades sociais na infância: Teoria e prática. (4.ed.). Petrópolis, RJ : Vozes. 
Dereli, E. (2009). Examining the permanence of the effect of a social skills training program for the acquisition of social problemsolving skills. Social Behavior and Personality, 37 (10), 14191428. doi: 10.2224/sbp.2009.37.10.1419

Dinnebeil, L. A., Sawyer, B. E., Logan, J., Dynia, J. M., Cancio, E., \& Justice, L. M. (2013). Influences on the congruence between parent's and teacher's ratings of Young children's social skills and problem behaviors. Early Childhood Research Quarterly, 28, 144-152.

Feitosa, F. B., Del Prette, Z. A. P., Del Prette, A., \& Loureiro, S. R. (2011). Explorando relações entre comportamento social e o desempenho acadêmico em crianças. Estudos e Pesquisas em Psicologia, 11(2), 442-455.

Fram, M. S., Kim, J., \& Sinha, S. (2012). Early care and prekindergarten care as influences on school readiness. Journal of Family Issues, 33(4), 478-505. doi: $1177 / 0192513 \times 11415354$

Fumo, V. M. S. (2009). Habilidades sociais acadêmicas de crianças com baixo e alto rendimento acadêmico na interação com o professor (Dissertação de mestrado, Universidade Federal de São Carlos, São Carlos).

Garaigordobil, M. \& Maganto, C. (2011). Empatía y resolución de conflictos durante la infancia y la adolescencia. Revista Latinoamericana de Psicologia, 43(2), 255-266.

Gresham, F. M. (2009). Análise do comportamento aplicada às habilidades sociais. In A., Del Prette \& Z. A. P., Del Prette (Orgs), Psicologia das Habilidades Sociais: Diversidade teórica e suas implicações (pp.17-66). Petrópolis, RJ: Vozes.

Leme, V. B. R., Del Prette, Z. A. P., Koller, S. H. \& Del Prette, A. (2015). Habilidades sociais e o modelo bioecológico do desenvolvimento humano: análise e perspectivas. Psicologia \& Sociedade, 1-13, doi: 10.1590/1807-03102015aop001

Marturano, E. M., \& Loureiro, S. R. (2007). O desenvolvimento socioemocional e as queixas escolares. In Del Prette, A. \& Del Prette, Z. A. P (Orgs). Habilidades Sociais, Desenvolvimento e aprendizagem. Questões conceituais, Avaliação e Intervenção. (pp.259-291). Campinas, SP. Ed. Alínea.

Moreira, P., Crusellas, L., Sá, I., Gomes, P., \& Matias, C. (2010). Evaluation of a manual-based programme for the promotion of social and emotional skills in elementary school children: results from a 4-year study in Portugal. Health Promotion International, 25(3), 309-317. doi: 10.1093/heapro/daq029

Moreira, P. A. S., Jacinto, S., Pinheiro, P., Patrício, A., Crusellas, L., Oliveira, J. T., \& Dias, A. (2014). Long-Term Impact of the Promotion of Social and Emotional Skills. Psicologia Reflexão e Crítica, 27(4), 634-641, doi: 10.1590/1678-7153.201427404 
Molina, R. C. M., \& Del Prette, Z.A P. (2006). Funcionalidade da relação entre habilidades sociais e dificuldades de aprendizagem. Psico-USF, 11(1), 53-63. doi: 10.1590/S141382712006000100007

Montroy, J. J., Bowles, R.P., Skibbe, L.E., \& Fostter, D. (2014). Social skills and problem behaviors as mediators of the relationship between behavioral self-regulation and academic achievement. Early Childhood Research Quarterly, 29, 298-309, http://dx.doi.org/10.1016/j.ecresq.2014.03.002

Pizato, E. C. G., Marturano, E. M., \& Fontaine, A. M. G. V. (2014). Trajetórias de Habilidades Sociais e Problemas de Comportamento no Ensino Fundamental: Influência da Educação Infantil. Psicologia: Reflexão e Crítica, 27(1), 189 197.

Sabol, T. J., \& Pianta, R. C. (2011). Patterns of school readiness forecast achievement and socioemotional development at the end of elementary school. Child Development, 00(0),1-18. doi: $10.1111 / \mathrm{j} .1467-8624.2011 .01678$.

Saud, L. F., \& Tonelotto, J. M. (2005). Comportamento social na escola: diferenças entre gênero e séries. Psicologia Escolar e Educacional, 9(1), 47-57.

Valle, T. G. M., \& Garnica, K. R. H. (2009). Avaliação e treinamento de habilidades sociais de crianças em idade pré-escolar. In T. G. M., Valle (Org). Aprendizagem e desenvolvimento humano: avaliações e intervenções (pp. 48-75). São Paulo: Cultura Acadêmica.

Welsh, M., Parke, R. D., Widaman, K., \& O'Neil, R. (2001). Linkages between children's social and academic competence: A longitudinal analysis. Journal of School Psychology, 39(6), 463482.

\section{Endereço para correspondência \\ Thaciana Araujo da Silva}

Universidade Federal do Pará - UFPA

Programa de Pós-graduação em Teoria e Pesquisa do Comportamento Rua Augusto Corrêa, 01, Guamá, CEP 66075-110, Belém - PA, Brasil Endereço eletrônico: thacianaaraujo@yahoo.com.br

\section{Lília I êda Chaves Cavalcante}

Universidade Federal do Pará - UFPA

Programa de Pós-graduação em Teoria e Pesquisa do Comportamento

Rua Augusto Corrêa, 01, Guamá, CEP 66075-110, Belém - PA, Brasil

Endereço eletrônico: liliaccavalcante@gmail.com

Recebido em: 23/5/2016

Reformulado em: 30/11/2016

Aceito em: $30 / 11 / 2016$ 


\section{Notas}

* Doutoranda e Mestre em Teoria e Pesquisa do Comportamento, UFPA.

** Doutora em Teoria e Pesquisa do Comportamento, UFPA, professora do Programa de Pós-Graduação em Teoria e Pesquisa do Comportamento, área de concentração da Ecoetologia. 